\title{
DYNAMICAL EVOLUTION OF A MODEL PLANETARY NEBULA
}

\author{
W. G. MatheWs \\ (University of California, San Diego, U.S.A.)
}

The gas-dynamical equations for conservation of mass, momentum and energy, the ionization equation, and the equation of transfer for the ionizing radiation have been solved in a manner which approximates conditions in planetary nebulae. The ionized and neutral gas are assumed to heat and cool according to a linearized rate proportional to

$$
\frac{3}{2} k\left(T-T_{\text {eq }}\right), \text { where } T_{\text {eq }}\left(\mathrm{H}_{\mathrm{II}}\right)=10^{4}{ }^{\circ} \mathrm{K} \text { and } T_{\text {eq }}\left(\mathrm{H}_{\mathrm{I}}\right)=500^{\circ} \mathrm{K} .
$$

The initial configuration is a spherical shell of mass $M_{n}=0.307 M_{\odot}$ with outer radius 0.027 parsec, thickness 0.002 parsec, temperature $500^{\circ} \mathrm{K}$, density $7.5 \times 10^{5}$ $\mathrm{cm}^{-3}$, and a uniform outward velocity of $20 \mathrm{~km} / \mathrm{sec}$. The luminosity, temperature, and surface gravity of the central star are assumed to vary as functions of the projected nebular radius according to the evolutionary scheme developed by Seaton and Harman. At each step in the calculation the projected radius of the nebula is determined and the ionizing photon luminosity at the inner edge of the nebula is therefore known. As in earlier models (Mathews, 1966), a dynamical pressure is found to be required to maintain the shell appearance of the nebula. The stellar wind which produces this dynamical pressure at the inner edge of the nebula $\left(R=R_{\mathrm{in}}\right)$ is proportional to $R_{\mathrm{in}}^{-2}\left[u_{0}^{2}-2\left(G M_{*} g\right)^{1 / 2}\right]^{1 / 2}$. Here a simple ballistic theory is used in which the gas leaves the stellar surface with a velocity $u_{0}=1000 \mathrm{~km} / \mathrm{sec}$. The central star of mass $M_{*}=1.0 M_{\odot}$ has a surface gravity $g$ which is calculated as a function of time from the projected radius. The dynamical pressure eventually drops to zero when $g \geqslant u_{0}^{4} /\left(4 G M_{*}\right)$.

As the gas at the inner edge begins to ionize, the pressure throughout the nebula is equalized by a shock which moves outward through the neutral gas. Later, when about $1 / 10$ of the nebular mass is ionized, a second shock is released from the ionization front, and this shock moves through the neutral shell reaching the outer edge after about 1400 years. The density in the Hi gas just behind this shock is quite large $\left(10^{6}-10^{5} \mathrm{~cm}^{-3}\right)$, and the outward gas velocity increases from within until it reaches a maximum of $40-80 \mathrm{~km} / \mathrm{sec}$ just behind the shock front. The projected appearance of the nebula during this stage has a double-ring structure similar to many observed planetaries. The most interesting development is an apparent shrinking of the (projected) nebula which occurs from 1200 to 1300 years, i.e. just after the nebula is completely ionized. This is caused by the large gas velocities in the outer parts of the shell which, when ionized, expand so fast into the external vacuum that an apparent 
inward-moving rarefaction wave results. During this same period the outward expansion velocity which would be observed at the centre of the nebula would be only $\sim 22 \mathrm{~km} / \mathrm{sec}$. The recent proper-motion observations of the Lillers, which show apparent still-stands or shrinkages, might well be caused by this effect. The projected radius at the instant when the nebula first becomes thin to the Lyman continuum is 0.068 parsec, in excellent agreement with the value given by Seaton and Harman. The subsequent evolution of the completely ionized nebula is very similar to the models calculated earlier (Mathews, 1966). A massive ejection followed by a stellar wind thus appears to explain many of the observed features of planetary nebulae.

\section{Reference}

Mathews, W.G. (1966) Astrophys. J., 143, 173.

\section{DISCUSSION}

Seaton: Is it possible to say what fraction of the energy absorbed from the central star goes into dynamical motion (the remainder being emitted as radiation of the nebula)?

Kahn: The fraction of the energy output of the star which goes into kinetic energy of motion is of the order of one or a few percent.

Savedoff: Is not the stellar wind introduced in your models just to maintain the central holes? It seems to me that if the condition of constant initial velocity were relaxed, one could construct other initial velocity fields which would maintain the central depression.

Williams: In a previous paper, you gave parameters for the stellar wind which were consistent with the values, $N \approx 1 \mathrm{~cm}^{-3}$, and $U_{+} \approx 550 \mathrm{~km} / \mathrm{sec}$. In view of the sharp peak in the density distribution in the present models, would you now change these numbers?

Mathews: The velocity of mass loss cannot be determined from these models. The dynamical pressure of the stellar wind depends on both the gas density and the square of the gas velocity just before shocking against the inner edge of the planetary shell. The earlier figures which I mentioned were only representative possibilities. 